OBESITY AND

MYOCARDIAL

APOPTOSIS

\title{
Impact of dietary-induced obesity on adrenergic-induced cardiomyocyte damage in rats
}

Leanda Vengethasamy*, Olebogeng H.I. Majane*,

Eugene F. du Toit", Gavin R. Norton* and Angela J. Woodiwiss*

"Cardiovascular Pathophysiology and Genomics Research Unit, School

of Physiology, Faculty of Health Sciences, University of the

Witwatersrand, Johannesburg

\#Department of Biomedical Sciences, Faculty of Health Sciences,

University of Stellenbosch, Tygerberg

Address for correspondence:

Angela Woodiwiss

Cardiovascular Pathophysiology and Genomics Research Unit

School of Physiology

University of the Witwatersrand Medical School

7 York Road

Parktown

2193

South Africa

Email:

angela.woodiwiss@wits.ac.za

\section{INTRODUCTION}

Obesity is a risk factor for heart failure independent of other conventional cardiovascular risk factors. ${ }^{(1-3)}$ As adjustments for cardiac systolic chamber function abolish the relationship between obesity and heart failure(2) it is possible that heart failure in obesity is caused by pump dysfunction rather than diastolic abnormalities. There is increasing evidence from both human ${ }^{(4,5)}$ and animal $^{(6-9)}$ studies that obesity is associated with myocardial contractile disturbances. These contractile disturbances may occur in even mild-to-moderate forms of excess adiposity. ${ }^{(4,5)}$ However, the mechanisms responsible for this effect remain uncertain.

An increased cardiomyocyte apoptosis occurs in genetic animal models of marked obesity ${ }^{(10,1)}$ - an effect that may in part contribute toward obesity associated reductions in myocardial contractile function. However, data obtained in genetic models of marked obesity may not be representative of the more common forms of mild-to-moderate obesity that are induced by dietary

\section{ABSTRACT}

Although obesity is an independent risk factor for heart failure and even mild-to-moderate forms of obesity are associated with myocardial systolic dysfunction the mechanisms of the myocardial dysfunction have not been identified. We assessed whether dietary-induced obesity is associated with an increased sensitivity of the myocardium to $\beta$-adrenergic-induced cardiomyocyte apoptosis or fibrosis. To induce obesity, rats were fed a diet that promotes an increased caloric intake. Adrenergic-induced cardiomyocyte apoptosis was determined by injecting rats for 5 days with isoproterenol $(0.01 \mathrm{mg} / \mathrm{kg} /$ day for 3 days and $0.02 \mathrm{mg} / \mathrm{kg} / \mathrm{day}$ for 2 days) and then studying the degree of cardiomyocyte damage using a TUNEL assay and assessing the pathological score. Five months of feeding rats a diet that promoted the development of an increased body weight (Control=48 I $\pm 4.3 \mathrm{~g}$, Diet $=550 \pm 7.8 \mathrm{~g}, \mathrm{p}<0.00 \mathrm{I})$ and visceral fat content (Control=19.6 $\pm 0.8 \mathrm{~g}$, Diet=33.0 $\pm \mathrm{I} .2 \mathrm{~g}, \mathrm{p}<0.000 \mathrm{I}$ ), did not alter baseline cardiomyocyte apoptosis. However, 5 days of $\beta$-adrenergic activation resulted in an enhanced cardiomyocyte apoptosis in rats receiving the experimental diet as compared to rats receiving a normal diet $(p<0.01)$. No changes in the myocardial pathological score (fibrosis) were noted. The enhanced adrenergic-induced cardiomyocyte apoptosis in obese rats could not be explained by dietaryinduced increases in baseline left ventricular internal diameters, decreases in systolic function (endocardial or midwall fractional shortening) or differences in the response of the heart to adrenergic-induced increases in inotropic or chronotropic function. In conclusion, the present study suggests that obesity may contribute to myocardial dysfunction by increasing the sensitivity of the myocardium to adrenergicinduced cardiomyocyte damage. SAHeart 2009; 6: 154-160

effects. Moreover, whether genetic models of marked obesity $(10,11)$ exhibit cardiomyocyte apoptosis independent of conventional risk factors such as hypertension or diabetes mellitus, is uncertain. ${ }^{(12-15)}$ We have recently developed an animal model of dietary-induced obesity without increases in blood pressure and with a normal blood glucose control,(16) but, in the present study we evaluated whether this animal model is associated with excessive cardiomyocyte apoptosis. Obesity is a risk factor for heart failure; (1-3) heart failure is associated with excessive adrenergic activation; ${ }^{(17)}$ 
and adrenergic activation is a well-recognised stimulus for cardiomyocyte apoptosis; ${ }^{(18)}$ we therefore also explored the possibility that dietary-induced obesity increases the susceptibility of the myocardium to $\beta$-adrenergic agonist-induced cardiomyocyte apoptosis.

\section{METHODS}

Study groups

The present study was approved by the University of the Witwatersrand Animal Ethics Screening Committee (approval numbers: 2006/59/04 and 2006/99/03) and complies with the National Institutes of Health Guide for the Care and Use of Laboratory Animals. Dietary-induced obesity was produced by feeding Wistar-Kyoto rats an experimental diet $(n=30)$ recently described ${ }^{(16)}$ which consists of $65 \%$ carbohydrate, 19\% protein, and $16 \%$ fat in comparison to that of a control diet $(n=30)$ which consists of $60 \%$ carbohydrate, $30 \%$ protein, and 10\% fat. As the experimental diet is designed to induce hyperphagia(16) the experimental groups consumed a greater quantity of food than the animals fed the control diet and, consequently, energy intake was enhanced in the experimental groups $(570 \pm 23 \mathrm{~kJ} /$ day $)$ compared to the control group that consumed $37 \mathrm{I} \pm 18 \mathrm{~kJ} /$ day. Our research groups have previously shown that differences in micronutrient (vitamins and minerals) intake produced by dilution of the diet by addition of carbohydrates and fats does not modify either body size or cardiac structure or function. ${ }^{(16)}$ After 5 months of feeding the different diets, rats were randomly assigned to receive either the $\beta$-adrenoreceptor agonist, isoproterenol (ISO) (0.01 $\mathrm{mg} / \mathrm{kg} /$ day for 3 days and $0.02 \mathrm{mg} / \mathrm{kg} /$ day for 2 days) ${ }^{(18)}$ (Diet-ISO, n= 14; Control-ISO, $n=14$ ) or the saline vehicle (DietSaline, $n=\mid 6$; Control-Saline, $n=16$ ). Isoproterenol was constituted in sterile saline $(0.9 \% \mathrm{NaCl})$ in order that each rat would receive subcutaneous injections of $\sim 0.1-0.2 \mathrm{ml}$ volumes daily. Control rats received daily injections of sterile saline at the same volume.

Blood pressures, blood glucose control and assessment of adiposity

Non-invasive tail cuff blood pressures ${ }^{(15)}$ were measured at the beginning and at the end of the study in all rats to confirm the previously described lack of effect of the experimental diet on blood pressure.(16) The percentage glycosylated haemoglobin $\left(\mathrm{HbA}_{\mid c}\right)$ was determined to confirm the previously described normal blood glucose control in the experimental diet fed rats. ${ }^{(16)}$ $\mathrm{HbA}_{\mathrm{Ic}}$ was determined in blood samples obtained from the thoracic cavity immediately after extirpation of the heart, using the HBAICll Tina-Quant kit (Cobas-Roche Diagnostics). Body weights were recorded in rats before the start of feeding and then once a month at the same time on the same day of each week until the end of the study. At the end of the study, visceral fat weight was determined post-mortem. To weigh visceral fat, fat was removed from the retroperitoneal space on the posterior wall of the abdominal cavity and from the omentum.

\section{Echocardiography}

At the end of the study left ventricular systolic function and chamber dimensions were determined in vivo as previously described ${ }^{(19)}$ in anaesthetised rats $(50 \mathrm{mg} / \mathrm{kg}$ of ketamine and $3 \mathrm{mg} /$ $\mathrm{kg}$ of xylazine) before and 15 minutes after an acute injection of isoproterenol at the doses administered to rats to induce cardiomyocyte apoptosis. In this component of the study 8 rats from each of the saline-treated groups and 10 rats from each of the ISO groups were randomly selected. Left ventricular enddiastolic (LVEDD) and end-systolic (LVESD) internal diameter and end-diastolic (ED PWT) and end-systolic posterior wall thickness (ES PWT) were measured with two-dimensional targeted $M$ mode echocardiography (7.5 MHz transducer and a Hewlett Packard Sonos 2500 sector scanner). Left ventricular chamber and myocardial systolic function were determined from endocardial (FSend) and midwall (FSmid) fractional shortening respectively, as previously described.(19) The following formulae were employed to determine FSend and FSmid:

$$
\begin{aligned}
& \text { FSend }=\text { EDD-ESD/EDD } \times 100 \\
& F S \text { mid }=\frac{[(E D D+E D P W T) / 2-(E S D+E S P W T) / 2]}{(E D D+E D ~ P W T) / 2} \times 100
\end{aligned}
$$

\section{Cardiomyocyte fibrosis and apoptosis}

After echocardiography in those rats in which echocardiography was performed (Diet-ISO, $n=10$; Diet-Saline, $n=8$; Control-ISO, $\mathrm{n}=10$; Control-Saline, $\mathrm{n}=8$ ), and in the remaining rats (Diet-ISO, $n=4$; Diet-Saline, $n=8$; Control-ISO, $n=4$; Control-Saline, $n=8$ ), the heart was removed from the thoracic cavity under anaesthesia and weighed. Left ventricular tissue was stored in formalin, processed routinely for light microscopy and $50 \mu \mathrm{m}$ thick sections of the long axis circumference cut through the full thickness of the left ventricular wall. Ten slices were obtained at I mm intervals and stained with van Gieson's stain. After staining, a pathological grade was assigned, where 0 indicates no damage; I and 2, patchy fibrosis in less than or more than $20 \%$ of the field respectively; 3 and 4 , diffuse contiguous subendocardial fibrosis in less than or more than $50 \%$ of the field respectively and 5 and 6 , full 
thickness fibrosis in less than or more than $50 \%$ of the field respectively. ${ }^{(20)}$

The degree of apoptosis was quantified as previously described $^{(18,21)}$ on $5 \mu \mathrm{m}$ thick myocardial tissue sections obtained from the same tissue blocks used to assess the pathological score. Nuclear deoxyribonucleic acid (DNA) fragments in the tissue sections were detected using a non-radioactive in situ apoptotic cell death detection kit (DeadEnd ${ }^{\mathrm{TM}}$ Colorimetric TUNEL system, Promega, Madison, WI, USA), where terminal deoxynucleotidy transferase (TdT) was used to incorporate biotinylated nucleotide at the 3'-OH DNA ends. The number of apoptotic cardiomyocyte nuclei and the total number of cardiomyocyte nuclei (haematoxylin and eosin stain) in each slide were counted on ten evenly spaced fields from the apex to the base using a computer-based image acquisition and analysis system at 400 times magnification (Axiovision 3, Carl Zeiss, Gottingen, Germany). Apoptotic cardiomyocyte nuclei were expressed as a percentage of the total number of cardiomyocyte nuclei.

\section{Data analysis}

All data are presented as mean \pm SEM. Comparisons between groups were made with a two-way ANOVA or with a Student's t-test when pooled analysis was performed.

\section{RESULTS}

\section{Measures of excess adiposity}

Figure I shows the effect of the experimental diet on body weight. The experimental diet resulted in a 15\% increase in body weight and a $74 \%$ increase in visceral fat weight (Control diet $=19.6 \pm 0.8 \mathrm{~g}$, Experimental diet $=33.0 \pm 1.2 \mathrm{~g}, \mathrm{p}<0.000 \mathrm{I}$ ) as compared to control diet fed rats. The body weight in rats receiving the experimental diet was statistically greater than the body weight in rats receiving the control diet, from the fourth month of the study (Figure I). Importantly, similar dietary effects on body weight and visceral fat were noted in rats assigned to receive either isoproterenol or the vehicle at the end of the study period (data not shown).

Blood pressures and percentage glycosylated haemoglobin Dietary-induced obesity had no effect on tail-cuff systolic blood pressure (Experimental diet: Baseline $=116 \pm 3 \mathrm{~mm} \mathrm{Hg}$ : After 5 months of the study $=1 \mid 8 \pm 4 \mathrm{~mm} \mathrm{Hg}$; Control diet: Baseline $=112$ $\pm 4 \mathrm{~mm} \mathrm{Hg}$ : After 5 months of the study $=119 \pm 4 \mathrm{~mm} \mathrm{Hg}$ ). In addition, the obesity-inducing diet did not influence the percen-

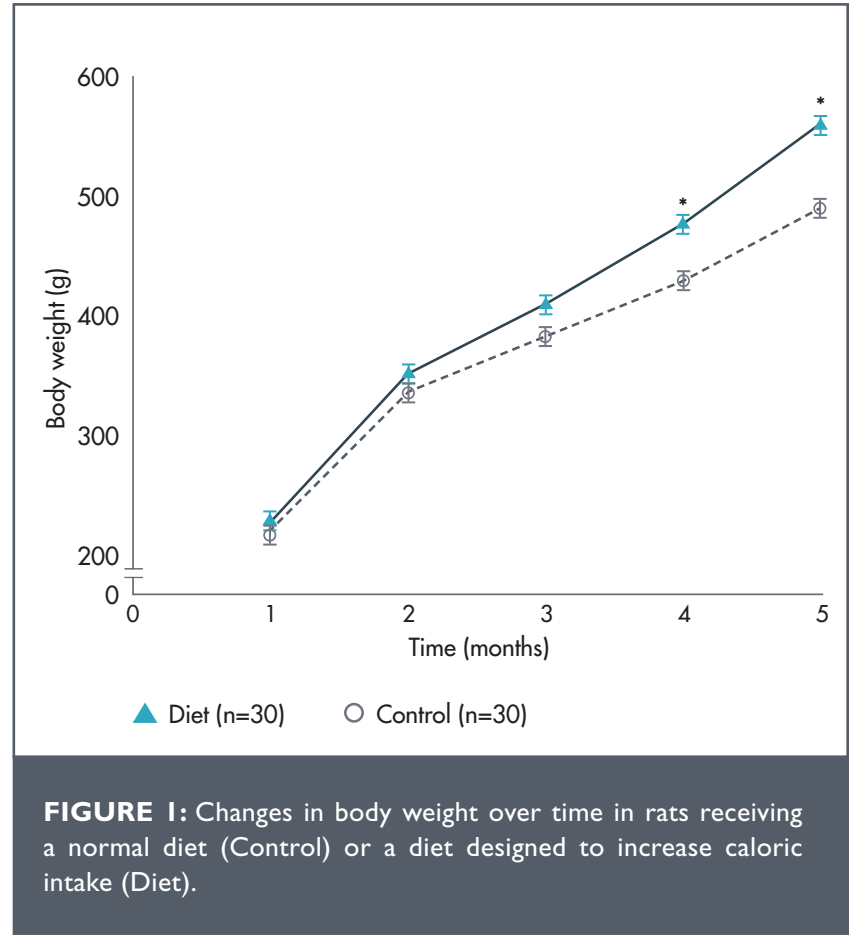

* $p<0.00$ I vs Control group

tage glycosylated haemoglobin (Experimental diet $=4.69 \pm 0.13 \%$, Control diet $=4.68 \pm 0.05 \%$ )

Impact of obesity and short-term adrenergic stimulation on heart weight

As compared to the control diet fed groups a trend for an increase in heart weight and left ventricular weight was noted in the experimental diet fed groups (Table I). In a pooled analysis of all control diet fed as compared to all experimental diet fed rats, the experimental diet resulted in an increase in heart weight (Experimental diet $=1.23 \pm 0.02[\mathrm{n}=30]$, Control diet $=1.14$ \pm 0.02 [ $n=30], p<0.005$ ) and left ventricular weight (Experimental diet $=1.01 \pm 0.02$, Control diet $=0.94 \pm 0.02, p<0.005$ ). Short term isoproterenol administration failed to modify heart weight or left ventricular weight in the control or the experimental diet groups as compared to their respective control groups (Table I). Moreover, isoproterenol failed to modify heart weight or left ventricular weight in pooled analysis comparing data obtained in all rats receiving isoproterenol versus all rats receiving the vehicle (data not shown). In a comparison of the four groups, left ventricular weight was greater in the experimental diet group receiving isoproterenol as compared to the two control diet fed groups (Table I). As the experimental diet resulted in a greater increase in body weight $(\sim 15 \%)$ as compared to heart $(\sim 8 \%)$ or left ventricular weight $(\sim 7 \%)$ a decrease in the heart and left ventri- 
cular weight-to-body weight ratios was noted in the experimental diet fed groups.

Impact of obesity and short-term adrenergic stimulation on left ventricular systolic function, heart rate and chamber dimensions

Figure 2 shows left ventricular systolic chamber and myocardial function and heart rate in rats at the end of the study and inotropic and chronotropic responses to acute administration of isoproterenol. Neither the experimental diet given for 5 months, nor 5 days of isoproterenol administration modified baseline left ventricular chamber or myocardial systolic function or heart rate. What is more, neither the experimental diet nor isoproterenol administration modified inotropic or chronotropic responses to acute isoproterenol administration.

In a pooled analysis of all experimental diet fed rats as compared to all control diet fed rats, the experimental diet tended to increase left ventricular wall thickness (Experimental diet $=1.96$ \pm 0.06 , Control diet $=1.83 \pm 0.04, p=0.07$ ) but did not modify left ventricular internal dimensions (Experimental diet $=8.54 \pm 0.12$, Control diet=8.44 $\pm 0.17, p=0.65$ ). Table 2 shows LV internal dimensions and wall thickness in the 4 groups of rats at the end of the study. Neither the experimental diet given for 5 months, nor 5 days of isoproterenol administration modified either LV internal dimensions or wall thickness.

Impact of obesity and isoproterenol on cardiomyocyte apoptosis and fibrosis

Figure 3 shows the effect of isoproterenol given for 5 days as well as the experimental diet on the percentage TUNEL positive stained cardiomyocyte nuclei and the pathological score of the left ventricle. The experimental diet alone failed to increase the

TABLE I: Cardiac morphometry in rats fed different diets for 5 months and subsequently receiving either isoproterenol (ISO) or the saline vehicle for 5 days.

\begin{tabular}{|l|lllll}
\hline & HW $(\mathrm{g})$ & LVW $(\mathrm{g})$ & HW/BW & LVW/BW \\
\hline Control-Saline $(n=16)$ & $1.13 \pm 0.02$ & $0.94 \pm 0.02$ & $2.35 \pm 0.05$ & $1.95 \pm 0.04$ \\
\hline Control-ISO $(n=14)$ & $1.15 \pm 0.03$ & $0.95 \pm 0.03$ & $2.39 \pm 0.06$ & $1.96 \pm 0.05$ \\
\hline Diet-Saline $(n=16)$ & $1.19 \pm 0.03$ & $0.98 \pm 0.02$ & $2.20 \pm 0.05$ & $1.82 \pm 0.04 * *$ \\
\hline Diet-ISO $(n=14)$ & $1.26 \pm 0.03$ & $1.04 \pm 0.02 *$ & $2.26 \pm 0.03$ & $1.85 \pm 0.02 * *$ \\
\hline
\end{tabular}

HW, heart weight; LVW, left ventricular weight; BW, body weight; Control, control diet; Diet, experimental diet. $* p<0.05$ versus both control diet fed groups; $* * 0<0.01$ versus respective control diet fed groups.
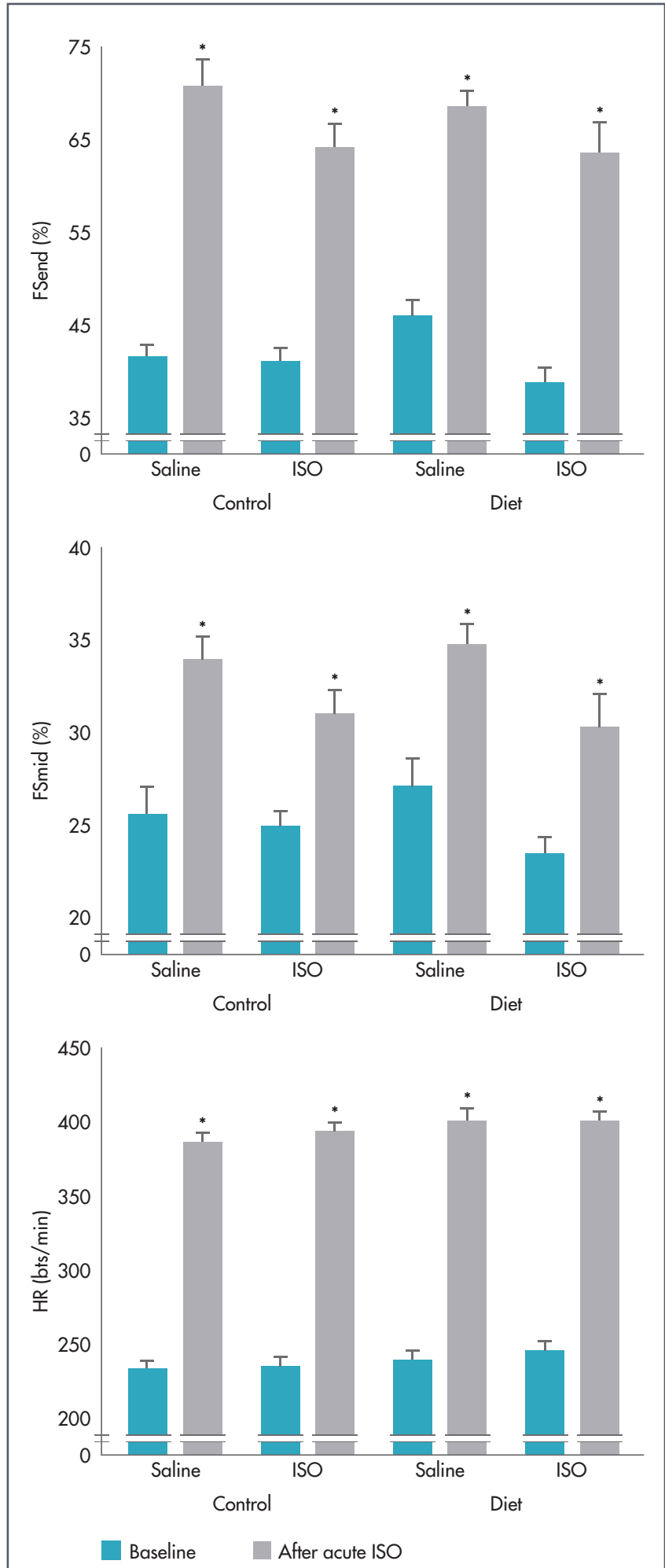

FIGURE 2: Effect of acute isoproterenol (ISO) administration on left ventricular systolic chamber (endocardial fractional shortening, FSend) and myocardial (midwall fractional shortening, FSmid) function as well as heart rate (HR) in rats receiving a normal diet (Control) or a diet designed to increase caloric intake (Diet). Saline, receiving the saline vehicle of ISO.

* $p<0.001$ vs baseline data. (Control-Saline, $n=8$; Control-ISO, $n=10$; Diet-Saline, $n=8$; Diet$150, n=10)$. 
TABLE 2: Comparison of left ventricular diameters and wall thickness values in rats fed different diets for 5 months and subsequently receiving either isoproterenol (ISO) or the saline vehicle for 5 days.

\begin{tabular}{lccccc}
\hline & $\begin{array}{c}\text { LVEDD } \\
(\mathbf{m m})\end{array}$ & $\begin{array}{c}\text { LVESD } \\
(\mathbf{m m})\end{array}$ & $\begin{array}{c}\text { PWTed } \\
(\mathbf{m m})\end{array}$ & $\begin{array}{c}\text { PWTes } \\
(\mathbf{m m})\end{array}$ \\
\hline Control-Saline $(n=8)$ & $8.46 \pm 0.18$ & $4.96 \pm 0.19$ & $1.87 \pm 0.06$ & $2.73 \pm 0.13$ \\
\hline Control-ISO $(n=10)$ & $8.43 \pm 0.28$ & $4.99 \pm 0.23$ & $1.79 \pm 0.06$ & $2.68 \pm 0.09$ \\
\hline Diet-Saline $(n=8)$ & $8.45 \pm 0.22$ & $4.59 \pm 0.24$ & $1.98 \pm 0.13$ & $3.02 \pm 0.14$ \\
\hline Diet-ISO $(n=10)$ & $8.61 \pm 0.12$ & $5.28 \pm 0.18$ & $1.96 \pm 0.06$ & $2.81 \pm 0.10$ \\
\hline
\end{tabular}

LVEDD, Left ventricular end diastolic diameter; LVESD, LV end systolic diameter; PWTed, LV end diastolic posterior wall thickness; PWTes, LV end systolic posterior wall thickness Control, control diet; Diet, experimental diet.

proportion of TUNEL positive stained cardiomyocyte nuclei. However, despite the inability of isoproterenol to significantly increase the proportion of TUNEL positive stained cardiomyocyte nuclei in control diet fed rats, rats fed the experimental diet developed an increase in the proportion of TUNEL positive stained cardiomyocyte nuclei as compared to all other groups. Importantly, neither the experimental diet nor isoproterenol affected the myocardial pathological score. Only minimal fibrosis (ranging from grades 0 to 3 ) was noted in each of the groups.

\section{DISCUSSION}

The main finding of the present study is that the myocardium of obese rats is more sensitive to the myocardial apoptotic effects that occur in response to adrenergic stimulation. The enhanced adrenergic-induced cardiomyocyte apoptosis in obese rats could not be explained by dietary-induced increases in baseline left ventricular internal diameters, decreases in systolic function (endocardial or midwall fractional shortening) or differences in the response of the heart to adrenergic-induced increases in inotropic or chronotropic function.

Previous studies have demonstrated that an increased cardiomyocyte apoptosis accompanies severe obesity in genetic animal models. ${ }^{(10,11)}$ In this regard, the present study supports the notion that obesity may promote cardiomyocyte apoptotic effects even in mild-to-moderate forms of obesity, albeit that this effect is only noted in the presence of an adrenergic stimulus. In the present study, the lack of ability of obesity per se to promote excessive cardiomyocyte apoptosis in the absence of an adrenergic stimulus may be accounted for by the relatively modest increase in body weight produced by the model studied (body weights $~ 15 \%$

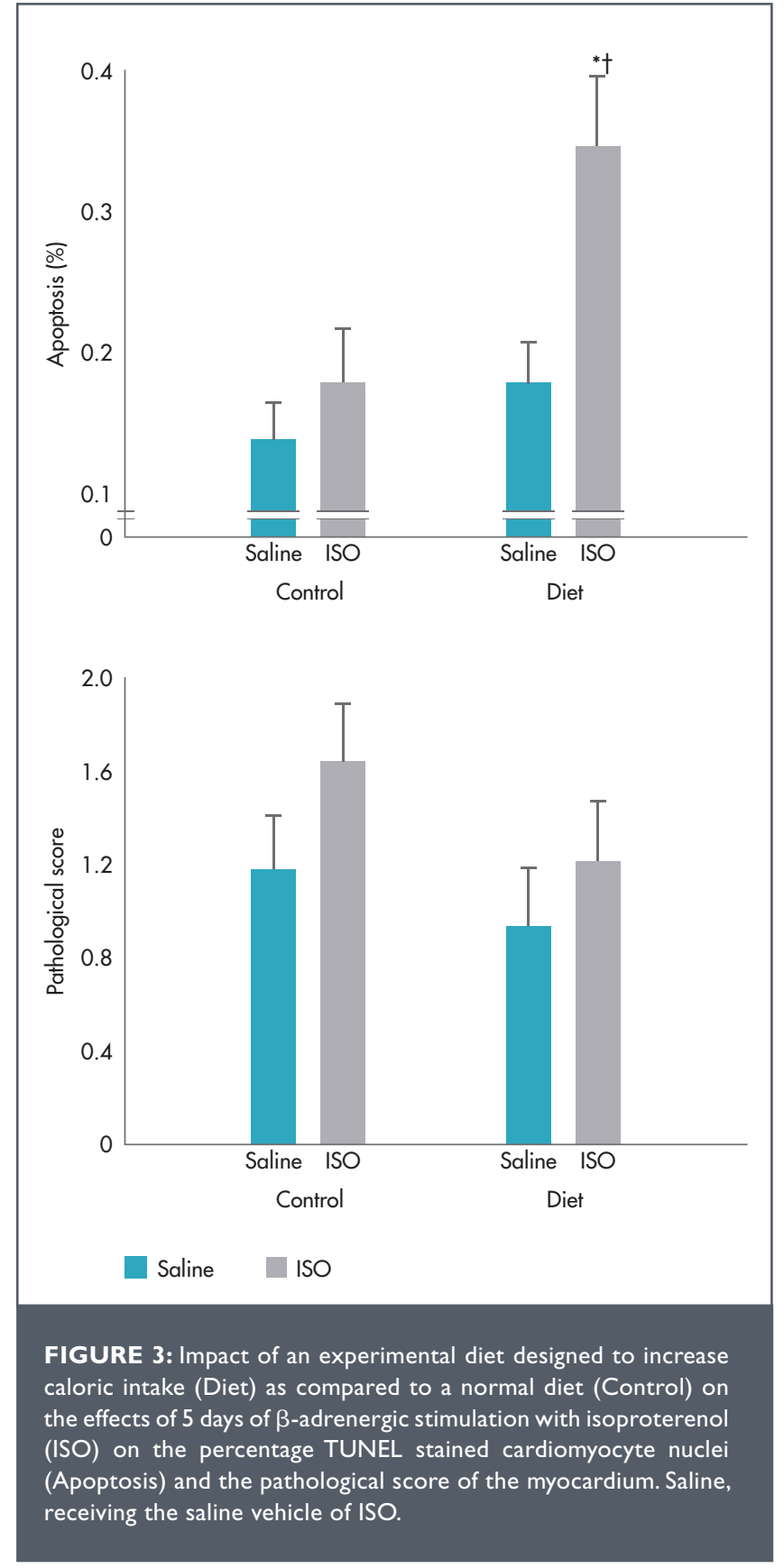

* $p<0.05$ versus Control-ISO, $+p<0.01$ vs Control-saline and Diet-saline groups. (ControlSaline, $n=16$; Control-ISO, $n=14$; Diet-Saline, $n=16$; Diet-ISO, $n=14$ ).

greater than lean animals). This is in contrast to previous studies reporting on obesity-induced effects on cardiomyocyte apoptosis in the absence of an exogenous stimulus such as a $\beta$-adrenoceptor agonist ${ }^{(10,11)}$ in genetic rodent models of obesity associated with considerably greater increases in body weights ( 45 to $84 \%$ greater than lean animals) $)^{(1,22)}$ than those reported on by us. In this regard however, the present study is more likely to reflect the predominant form of obesity that occurs at a population level (mild-to-moderate) in contrast to previous animal studies 
that are more likely to reflect the impact of more severe forms of obesity on the myocardium. ${ }^{(10,1)}$

In contrast to previous studies $(10,1)$ where it is uncertain whether the obesity-associated cardiomyocyte apoptotic effect occurs independently of alternative conventional risk factors such as hypertension or diabetes mellitus, ${ }^{(12-15)}$ in the present study we show that obesity is associated with adrenergic-induced cardiomyocyte apoptosis in a model without increases in blood pressure or alterations in blood glucose control (glycosylated haemoglobin) and in a model which we have previously reported to be euglycaemic.(16) Thus, the present study is the first to show that mild-to-moderate adiposity promotes cardiomyocyte apoptosis independent of blood pressure or blood glucose control. Importantly, however, this effect requires excessive adrenergic activation, a change that often accompanies obesity. ${ }^{(23)}$

In the present study the mechanisms of the increased susceptibility of the myocardium in obese rats to adrenergic-induced cardiomyocyte apoptosis were not identified. However, a few possibilities were considered. First, as the degree of obesity produced in rats in the present study promoted mild increases in left ventricular mass, it is possible that cardiac hypertrophy, which is a well-recognised risk factor for cardiomyocyte apoptosis, ${ }^{(24)}$ can explain the obesity-associated increase in adrenergic-induced cardiomyocyte apoptosis. Second, increases in myocardial reactive oxygen species, previously shown to mediate cardiomyocyte apoptosis(25) and produced by increments in cardiac wall stress need to be considered. In this regard, although we did not measure myocardial wall stress, it is unlikely that this mechanism plays a significant role, as resting systolic blood pressures and left ventricular chamber dimensions were unchanged, and adrenergic-induced inotropic and chronotropic responses in obese rats were comparable to those of lean rats. However, as myocardial $\beta$-adrenergic stimulation promotes the generation of reactive oxygen species, ${ }^{(26)}$ further studies are required to assess whether obese rat hearts are more susceptible to adrenergicinduced increases in the production or effects of reactive oxygen species. Importantly, it is unlikely that the increased adrenergicinduced cardiomyocyte apoptosis in obese rats can be explained by a combination of exogenous adrenergic actions and increased endogenous sympathetic effects, as this model of obesity is not associated with increases in endogenous norepinephrine release. ${ }^{(16)}$ Third, in genetic animal models, obesity-induced cardiomyocyte apoptosis may also be mediated by the accumulation of triglycerides in the heart.(II) In this regard, a combination of peri- pheral lipolysis induced by catecholamines and a decrease in the expression of myocardial enzymes responsible for fatty acid oxidation is thought to produce this effect. ${ }^{(11)}$ It is therefore possible that in the present study, obesity promoted a reduction in myocardial fatty acid oxidation, whilst the exogenous adrenergic stimulus produced lipolysis, the overall effect being an increase in cardiomyocyte apoptosis. Further work is required to explore this possibility.

Although caution should be exercised in translating these data to the clinical scenario, some possible clinical implications can be considered. As obesity is a risk factor for heart failure ${ }^{(1-3)}$ and heart failure is associated with excessive adrenergic activation ${ }^{(17)}$ it is important to consider the combination of these effects. The present data suggest that this combination mediates a synergistic effect to promote cardiomyocyte apoptosis. As cardiomyocyte apoptosis may have a causal role to play in the pathophysiology of ventricular dysfunction and its progression to cardiac failure, ${ }^{(27,28)}$ the present data suggest that myocardial decompensation in obese patients with heart failure may progress more rapidly than in lean patients unless appropriate adrenergic blockade is employed.

The limitations of the present study are as follows. First, the modest degree of obesity produced may have limited the outcomes of the present study. However, employing a model of modest as opposed to severe obesity is nevertheless entirely consistent with the high prevalence of mild-to-moderate obesity which presently exists world-wide, whilst more severe forms of obesity are less common. Further, by studying a more modest model of obesity, we biased the outcomes against ourselves, thus rendering our ability to show an impact of obesity on cardiomyocyte apoptosis even more impressive. Second the technique used to detect cardiomyocyte apoptosis (TUNEL technique) may overestimate the number of apoptotic nuclei, as it labels both DNA fragmentation and cells undergoing DNA repair. Alternative techniques such as the assessment of myocardial caspase-3 activity or other methods could have been employed to support the present outcomes. However, the assessment of myocardial caspase-3 activity requires freeze clamped tissue, the collection of which would have precluded the assessment of heart and left ventricular weights and the histological evaluation of apoptosis.

In conclusion, the results of the present study indicate that mildto-moderate obesity is associated with an increased susceptibility 
of the myocardium to adrenergic-induced cardiomyocyte apoptosis independent of changes in blood pressure, left ventricular dilatation or alterations in adrenergic-induced inotropic and chronotropic responsiveness. These findings cast further light on the mechanisms that may explain the independent relationship between obesity and the development of heart failure $\mathrm{e}^{(1-3)}$ and suggest that obesity may hasten the progression of cardiac decompensation in heart failure unless appropriate adrenergic blockade is employed. Clinical studies are required, conducted in obese and lean patients with comparable degrees of cardiac dysfunction and sympathetic activation at baseline, to address this issue.

\section{ACKNOWLEDGEMENTS}

This work was supported by the University Research Council of the University of the Witwatersrand, and the South African National Research Foundation (AJW and OHIM). LV was a recipient of a South African Medical Research Council scholarship in support of her higher degree.

\section{REFERENCES}

I. Kenchaiah S, Evans JC, Levy D, et al. Obesity and the risk of heart failure. New Engl J Med. 2002;347:305-3। 3.

2. Bahrami J, Bluemke DA, Kronwal R, et al. Novel metabolic risk factors for incident heart failure and their relationship with obesity. J Am Coll Cardiol. 2008;5।:1775-1783

3. Ingelsson E, Sundstrom J, Arnlov J, et al. Insulin resistance and risk of congestive heart failure. J Am Med Ass. 2005;294:334-34I.

4. Peterson LR, Waggoner AD, Schechtmann KB, et al. Alterations in left ventricular structure and function in young healthy obese women. J Am Coll Cardiol. 2004:43:1399-1404

5. Wong CY, O'Moore-Sullivan T, Leano R, et al. Alterations of left ventricular myocardial characteristics associated with obesity. Circulation. 2004; I 1 0:308 I-3087.

6. Caroll JF, Jones AE, Hester RL, et al. Reduced cardiac contractile responsiveness to isoproterenol in obese rabbits. Hypertension. 1997;30:1376-1381.

7. Relling DP, Esberg LB, Fang CX, et al. High-fat diet induced juvenile obesity leads to cardiomyocyte dysfunction and upregulation of Foxo3a transcription factor independent of lipotoxicity and apoptosis. J Hypertens. 2006;24:549-56।.

8. Dong $F$, Zhang $X$, Yang $X$, et al. Impaired cardiac contractile function in ventricular myocytes from leptin deficient ob/ob obese mice. J Endocrinology. 2006; 188:25-36

9. Ren J, Sowers JR, Walsh MF, et al. Reduced contractile response to insulin and IGF-I in ventricular myocytes from genetically obese Zucker rats. Am J Physiol. 2000;279:HI708-HI7|4.

10. Barouch LA, Gao D, Miller KL, et al. Cardiac myocyte apoptosis is associated with increased DNA damage and decreased survival in murine models of obesity. Circ Res. 2006;98:1 19-124.
11. Zhou Y-T, Grayburn P, Karim A, et al. Lipotoxic heart disease in obese rats: Implications for human obesity. Proc Nat Acad Sci. 2000;97: 1784- 1789.

12. Alonso-Galicia M, Brands MW, Zappe DH, et al. Hypertension in obese Zucker rats. Role of angiotensin II and adrenergic activity. Hypertension. 1996;28: 1047-1054.

13. Mazumder PK, O'Neill BT, Roberts MW, et al. Impaired cardiac efficiency and increased fatty acid oxidation in insulin-resistant ob/ob mouse hearts. Diabetes. 2004; 53:2366-2374.

14. Erickson JC, Hollopeter G, Palmiter RD. Attenuation of the obesity syndrome of ob/ob mice by the loss of neuropeptide Y. Science. 1996;274: 1704- 1707.

15. Corsettu JP, Sparks JD, Peterson RG, et al. Effect of dietary fat on the development of non-insulin dependent diabetes mellitus in obese Zucker diabetic fatty male and female rats. Atherosclerosis. 2000; 148:2431-24I.

16. du Toit EF, Smith W, Muller C, et al. Myocardial susceptibility to ischaemicreperfusion injury in a prediabetic model of dietary-induced obesity. Am J Physiol. 2008;294:H2336-H2343.

17. Anand IS, Fisher LD, Chiang YT, et al. Changes in brain natriuretic peptide and norepinephrine over time and mortality and morbidity in the Valsartan Heart Failure Trial (Val-HeFT). Circulation. 2003; 107:1278- 283.

18. Osadchii OE, Norton GR, McKechnie R, et al. Cardiac dilatation and pump dysfunction without intrinsic myocardial systolic failure following chronic $\beta$-adrenoreceptor activation. Am J Physiol. 2007;292:HI898-HI905.

19. Norton GR, Woodiwiss AJ, Gaasch WH, et al. Heart failure in pressure overload hypertrophy: The relative roles of ventricular remodeling and myocardial dysfunction. J Am Coll Cardiol. 2002;39:664-67।.

20. Tsotetsi OJ, Woodiwiss AJ, Netjhardt M, et al. Attenuation of cardiac failure, dilatation, damage, and detrimental interstitial remodelling without regression of hypertrophy in hypertensive rats. Hypertension. 200 I;38: 846-85I.

21. Veliotes DGA, Woodiwiss AJ, Deftereos DAJ, et al. Aldosterone receptor blockade prevents the transition to cardiac pump dysfunction induced by $\beta$-adrenoreceptor activation. Hypertension. 2005;45:9|4-920.

22. Mark AL, Shaffer RA, Correia MLG, et al. Contrasting blood pressure effects of obesity in leptin-deficient ob/ob mice and agouti yellow obese mice. J Hypertens. 1999:17:1949-1953.

23. Tentolouris N, Liatis $\mathrm{S}$, Katsilambros $\mathrm{N}$. Sympathetic system activity in obesity and metabolic syndrome. Ann NY Acad Sci. 2006; 1083:129-152.

24. Yamamoto S, Sawada K, Shimomura H, et al. On the nature of cell death during remodelling of hypertrophied human myocardium. J Mol Cell Cardiol. 2000;32: 161-175.

25. Siwik DA, Tzortzis JD, Pimental DR, et al. Inhibition of copper-zinc superoxide dismutase induces cell growth, hypertrophic phenotype, and apoptosis in neonatal rat cardiac myocytes in vitro. Circ Res. 1999;85: | 47- 153.

26. Zhang G, Kimura S, Nishiyama A, et al. Cardiac oxidative stress in acute and chronic isoproterenol- infused rats. Cardiovasc Res. 2005;65:230-238.

27. Abbate A, Biondi-Zoccai GGL, Bussani R, et al. Increased myocardial apoptosis in patients with unfavourable left ventricular remodelling and early symptomatic post-infarction heart failure. J Am Coll Cardiol. 2003;41:753-760.

28. Kocher AA, Schuster MD, Szabolcs MJ, et al. Neovascularisation of ischaemic myocardium by human bone-marrow-derived angioblasts prevents cardiomyocyte apoptosis, reduces remodelling and improves cardiac function. Nature Medicine. 2001;7:430-436. 